\title{
Mitochondrial function in vitrified versus slow-frozen murine embryos
}

\author{
Razif Dasiman a, b, Mimi-Sophia Sarbandi a, Nor-Shahida Abdul Rahman a, Salina Othman a, \\ Mastura Malek a, Fathiah Abdullah ${ }^{a}$, Fatin Nadzirah Zakaria ${ }^{b}$, Nina Keterina Hashim ${ }^{b}$, Nor- \\ Ashikin Mohamed Noor Khan ${ }^{a}{ }^{\star}$
}

a Maternofetal and Embryo Research Group (MatE), Faculty of Medicine, Universiti Teknologi MARA, Selangor Branch, Sungai Buloh Campus, 47000 Sungai Buloh, Selangor, Malaysia

${ }^{b}$ Faculty of Health Sciences, Universiti Teknologi MARA, Selangor Branch, Puncak Alam Campus, 42300 Puncak Alam, Selangor, Malaysia

*Corresponding author: noras011@salam.uitm.edu.my

\section{Article history}

Received 20 March 2018

Revised 5 April 2018

Accepted 8 May 2018

Published Online 14 April 2019

\begin{abstract}
The effects of vitrification and slow-freezing on mitochondrial functions of in vitro produced murine embryos at various developmental stages were investigated using the Confocal Laser Scanning Microscope (CLSM). Oocytes were obtained from superovulated females, fertilized with sperm and cultured. Resulting 2-, 4- and 8-cell embryos were collected and cryopreserved by vitrification and slow-freezing. Mitochondria were stained with MitoTracker Red (CMXRos). Images were viewed by CLSM and analyzed using QWin SoftwareV.3. Fluorescent intensities were used to indicate viability. Results showed that mitochondrial fluorescence intensities of cryopreserved embryos were significantly lower as compared to non-cryopreserved embryos $(p<0.01)$. Vitrification was found to be superior to slow-freezing at all developmental stages, based on mitochondrial function.
\end{abstract}

Keywords: Mitochondria, slow freezing, vitrification, murine embryos, Confocal Laser Scanning Microscope (CLSM)

(C) 2019 Penerbit UTM Press. All rights reserved

\section{INTRODUCTION}

Mitochondria are maternally inherited, and their numbers remain relatively constant in the embryo during the preimplantation stages as replication begins after implantation (Harvey et al., 2007). Apart from providing cellular energy, mitochondria have other essential roles in the regulation of programmed cell death and ions. They are therefore critical in the maintenance of viability and vitality of the embryo.

Emerging studies have demonstrated that mitochondria generate necessary energy ATP to perform dynamic polymerization and precision attachment of spindle fibers for cell differentiation and proliferation (Van Blerkom et al., 2000; Ludwig et al., 2001; Suzuki et al., 2006; Zeng et al., 2007). According to Takeuchi et al. (2005), mitochondria display specific patterns, and their distribution can be influenced by oxidizing agents, chemical or physical disturbances (Nagai et al., 2006; Dalcin at al., 2013). Cytometric assessment of mitochondria using fluorescent probes is useful to indicate mitochondrial function. In this study, MitoTrackerß Red was used as an indicator of mitochondrial and embryo viability. It is a sensitive indicator of relative changes in the mitochondrial membrane potential (MMP).

Over the years, cryopreservation has been extensively practiced in ART programmes where it functions to store excess gametes and embryos to achieve a high rate of pregnancy (Wennerholm, 2000; Saragusty and Arav, 2011; Dasiman et al., 2013). Two common cryopreservation techniques that have been applied extensively in mammalian embryology are ultra-rapid vitrification (Kasai, 2002; Lieberman et al., 2002), and conventional slow-freezing (Faten et al., 2010). Conventional slow-freezing was first introduced by Whittingham et al. (1972). It involves slow cooling of embryos using a programmable freezer before transfer for storage in liquid nitrogen at $-196^{\circ} \mathrm{C}$. Vitrification was discovered by Rall and Fahy (1985) who developed a cryoprotectant which formed transparent amorphous glass-like state while retaining its physical characteristics at $-196^{\circ} \mathrm{C}$.

The current study aims to compare the effects of vitrification and slow-freezing on mitochondrial of preimplantation embryos. Comparisons were made between mitochondrial intensities and distributions in 2-, 4- and 8-cell embryos, which were cryopreserved by vitrification and slow-freezing.

\section{EXPERIMENTAL}

\section{Materials and method}

Fifty female ICR mice aged 4-6 weeks old weighed 25-30 grams were used in this study. The experiment was performed in line with the ethical approval from the University Committee on Animal Research and Ethics (CARE) (Code: ACUC-7/11). To obtain in vitro fertilized embryos, the female mice were intraperitoneally superovulated using Pregnant Mare's Serum Gonadotropin (PMSG), and human Chorionic Gonadotropin (hCG) (5 IU/kg b.w) [Intervet, Holland] after 48 hours. Oocytes were collected from excised oviducts and washed with the M2 medium before being transferred into M16 droplets covered with mineral oil. Sperm was collected from the cauda epididymides of fertile males and transferred to the dish containing oocytes. Fertilized ova were observed daily under the inverted microscope [Olympus 1X81 SF-3, Japan]. Good quality 2cell embryos which had an intact zona pellucida and symmetrical blastomeres were selected for culture to the 4- and 8-cell stage.

Vitrification protocol was based on the methods by Nor-Ashikin (2006) and Nagy et al. (2003), while slow-freezing was based on Nagy et al. (2003). Embryos were cryopreserved at the 2-, 4- and 8cell stages of development. Prior to immunofluorescence staining, the 
embryos were warmed and fixed. The immunofluorescence staining method was based on Nor-Shahida et al. (2009). Nuclei were stained with 4', 6-diamino-2-phenylindole dihydrochloride (DAPI), permeabilized with $1 \%$ Triton-X in Phosphate Buffer Saline (PBS) and labeled with MitoTracker Red. Embryos were then counterstained with DAPI, mounted on slides and stored in the dark at $4^{\circ} \mathrm{C}$ overnight. Images taken from CLSM [Leica TCS SP5 AOBS, Germany] were converted to JPEG format before being analyzed using QWin Software V.3.The statistical analysis was performed using Statistical Package for Social Sciences Version 16 (SPSS Inc, USA). One-way analysis of variance (ANOVA) with Bonferroni correction was used, with statistical significance at $\mathrm{p}<0.05$.

\section{RESULTS AND DISCUSSION}

The confocal micrographs from Figure 1(a-i) shows the distributions of mitochondria in the 2-, 4-, and 8-cell stages of in vitro fertilized embryos. Mitochondria of the control group (noncryopreserved) embryos were distributed in the perinuclear region of blastomeres (Figures 1a, 1b, and 1c). Accumulation of mitochondria occured around the cell cortex. The blastomeres were uniform in size, with apically located nuclei.

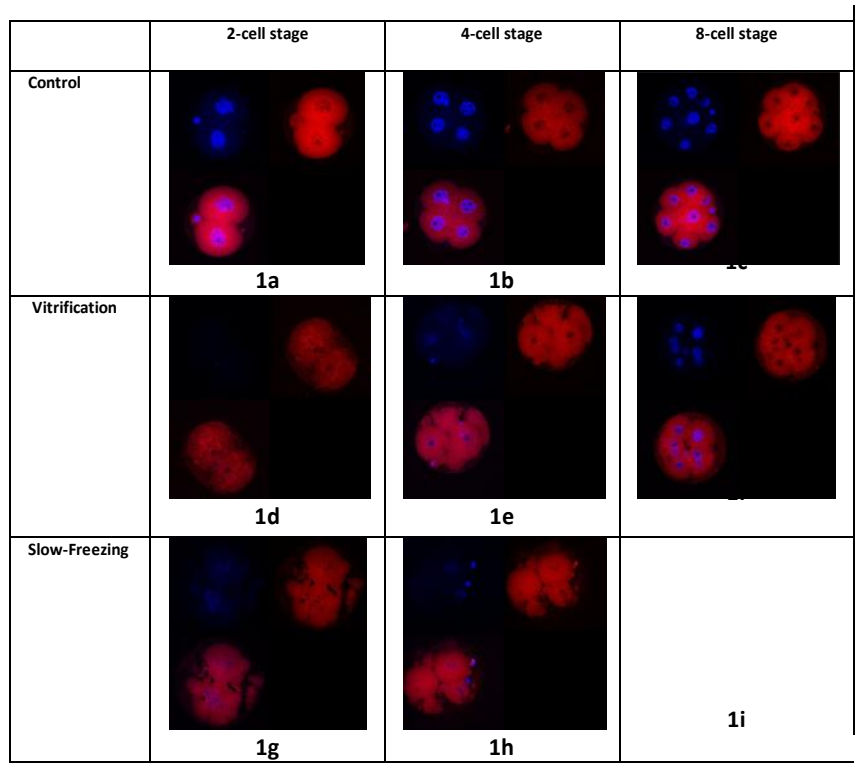

Figure 1(a-i) Mitochondrial distributions of 2-, 4- and 8-cell in vitro fertilized murine embryos. Control (1a, 1b, 1c), vitrified (1d, 1e, 1f), and slow-frozen $(1 \mathrm{~g}, 1 \mathrm{~h}, 1 \mathrm{i})$ embryos stained with MitoTracker Red and observed under CSLM. Blue DAPI staining represents nuclei.

In the vitrified group, damage to the nucleus and blastomeres were distinctly observed in 2- and 4-cell stage embryos. Blastomeres at these stages were uneven in size, compared to the 8-cell stage (Figures 1d, 1e, and 1f). Vitrified embryos displayed partial migration of mitochondria into the subcortical region. This was clearly observed in 2-cell embryos (Figure 1d). Vitrified 8-cell embryos showed good morphology with evenly-sized blastomeres, and fewer migration of mitochondria (Figure 1f).

Embryos which were slow-frozen displayed clustering and swelling of mitochondria and reduced fluorescence intensities (Figures 1g, 1h, and 1i). Blastomeres of the 2-, 4- and 8-cell stages embryos were not smooth and morphologically altered. Partial migration of the mitochondria was observed in both 2- and 4-cell embryos (Figures $1 \mathrm{~g}$ and $1 \mathrm{~h}$ ). Apart from that, fragmented and dissolved nucleus, cracked blastomeres and dislocation of mitochondria were also observed in all slow-frozen stages (Figures $1 \mathrm{~g}, 1 \mathrm{~h}$, and 1i). Mitochondrial diffusion into the subcortical region were significantly higher in vitrified 2-cell and slow-frozen 4-cell embryos as compared to the other groups and stages (Figure 1d and 1h). As mitochondria control the local concentrations of second messengers, mitochondrial translocation to discrete subcellular regions may contribute to signaling function prior to apoptosis (Al-Mehdi et al., 2012; Dewitt et al., 2006).

Table 1 Fluorescence intensities of mitochondrial in 2-, 4-, and 8-cell in vitro mouse embryos.

\begin{tabular}{cccc}
\hline $\begin{array}{c}\text { Freezing } \\
\text { techniques }\end{array}$ & $\begin{array}{c}\text { 2-cell } \\
(\mathrm{n}=50)\end{array}$ & $\begin{array}{c}\text { Fluorescence intensity }\left(\mathrm{x} 10^{5} \text { pixel }\right) \\
(\mathrm{n}=50)\end{array}$ & $\begin{array}{c}8 \text {-cell } \\
(\mathrm{n}=50)\end{array}$ \\
Control & $5.1 \pm 0.1^{\mathrm{a}}$ & $10.3 \pm 0.2^{\mathrm{a}}$ & $13.2 \pm 0.4^{\mathrm{a}}$ \\
Vitrified & $3.1 \pm 0.1^{\mathrm{b}}$ & $7.1 \pm 0.3^{\mathrm{b}}$ & $8.8 \pm 0.3^{\mathrm{b}}$ \\
$\begin{array}{c}\text { Slow } \\
\text { Freezing }\end{array}$ & $1.4 \pm 0.1^{\mathrm{c}}$ & $4.0 \pm 0.2^{\mathrm{c}}$ & $4.5 \pm 0.1^{\mathrm{c}}$ \\
\hline Values with different superscripts within the same column are significantly different $(p<0.01)$
\end{tabular}

For all stages of development, mitochondrial intensities were significantly higher in the vitrified group, compared to the slowfrozen group $(p<0.01)$. This indicated that mitochondrial function, and viability was better in vitrified embryos compared to slow-frozen embryos. In human studies, vitrification was also found to be more efficient than slow freezing for the cryopreservation of cleavage stage embryos (Valojerdi et al., 2009). The authors noted that vitrification resulted in higher survival rate, with minimal deleterious effects on post-warming embryo morphology.

\section{CONCLUSION}

Vitrification was found to be superior to slow-freezing at 2-, 4and 8-cell preimplantation stages, based on mitochondrial function.

\section{ACKNOWLEDGEMENT}

This research was funded by Ministry of Higher Education RAGS Grant (600-RMI/RAGS 5/3 (60/2015) and Universiti Teknologi MARA LESTARI Grant (No: 600-IRMI/MYRA 5/3/LESTARI (059/2017). The authors acknowledge all staff of the Institute of Medical Molecular Biotechnology (IMMB) and the Laboratory Animal Care Unit (LACU), UiTM Sg Buloh Campus, for their contribution and assistance throughout this study.

\section{REFERENCES}

Al-Mehdi, A-. B., Pastukh, V. M., Swiger, B. M., Reed, D. J., Patel, M R., Bardwell, G. C. Pastukh, V. V., Alexeyev, M. F., \& Gillespie, M. N. (2012). Perinuclear mitochondrial clustering creates an oxidant-rich nuclear domain required for hypoxia-induced transcription. Science Signaling, 5(231), 1-20.

Dalcin, L., Silva, R. C., Paulini, F., Silva, B. D. M., Neves, J. P., \& Lucci, C. M. (2013). Cytoskeleton structure, the pattern of mitochondrial activity and ultrastructure of frozen or vitrified sheep embryos. Cryobiology, 67(2), 137-145.

Dasiman, R., Nor-Shahida, A. R., Salina, O., Mohd-Fazirul, M., Norhazlin, J. M. Y., Wan-Hafizah, W. J., Mohd-Hamim, R., Gabriele, R. A. F., \& NorAshikin, M. N. K. (2013). Cytoskeletal alterations in different developmental stages of in vivo cryopreserved preimplantation murine embryos. Medical Science Monitor Basic Research, 19, 258-266.

Dewitt, D. A., Hurd, J. A., Fox, N., Townsend, B. E., Griffioen, K. J. S., Ghribi, O., \& Savory, J. (2006). Peri-nuclear clustering of mitochondria is triggered during aluminum maltolate induced apoptosis. Faculty Publications and Presentations, Paper 11.

Faten, F. A., Nina, D., Ahmed, M. A.S, Falcone, T., \& James, G. (2010). Slow freezing, vitrification and ultra-rapid freezing of human embryos: A systematic review and meta-analysis. Reproductive Biomedicine Online, 20, 209-222.

Harvey, A., Gibson, T., Quebedeaux, T., \& Brenner, C. (2007). Impact of assisted reproductive technologies: A mitochondrial perspective of cytoplasmic transplantation. Current Top Development Biology, 77, 229-249.

Kasai, M. (2002). Advances in the cryopreservation of mammalian oocytes and embryos: Development of ultrarapid vitrification. Reproductive Medicine and Biology, 1, 1-9. 
Lieberman, J., Isachenko, V., Rahimi, G., \& Tucker, M.J. (2002). The potential importance of vitrification in reproductive medicine. Biology Reproduction, $67,1671-1680$

Ludwig, T.E., Squirell, J.M., Palmenberg, A.C., \& Bavister, B.D. (2001). The relationship between development, metabolism and mitochondrial organization in 2-cell hamster embryos in the presence of low level of phosphates. Biology Reproduction, 65, 1648-1654.

Nagai, S., Mabuchi, H., Hirata, S., Kasai, T., \& Hoshi, K. (2006). Correlation of abnormal mitochondrial distribution in mouse oocytes with reduced developmental competence. Tohoku Japan Experimental Medicine, 210 137-144.

Nagy A., Gertsensten M., Vintersten K. and Behringer R. (2003). Manipulating the mouse embryo: A laboratory manual.(3rd edition). Cold Spring Harbor Laboratory Press, New York.

Nor Ashikin, M. N.K. (2006). In vitro and in vivo development of preimplantation murine embryos following gender identification using the polymerase chain reaction (PCR) technique. Ph.D. Thesis, University of Malaya.

Nor-Shahida, A. R., Dasiman, R., Salina, O., Gabrielle, R. A. F., \& Nor-Ashikin, M. N. K. (2009). Immunofluorescence staining of the murine preimplantation embryos: DAPI vs. Hoechst 33342. Proceedings of the $18^{\text {th }}$ Scientific Conference of Electron Microscopy Society of Malaysia (EMSM), Kuala Lumpur. 2009.

Rall, W. F. \& Fahy, G. M. (1985) Ice-free cryopreservation of mouse embryos at -196 degrees $\mathrm{C}$ by vitrification. Nature, $313,573-575$.
Saragusty, J., \& Arav, A. (2011). Current progress in oocyte and embryo cryopreservation by slow freezing and vitrification. Reproduction, 141(1), 1-19.

Suzuki, H., Satoh, M., \& Kabashima, K. (2006). Distributions of mitochondria and the cytoskeleton in hamster embryos developed in vivo and in vitro. Journal Mammalian Ova Research, 23, 128-134.

Takeuchi, T., Neri, Q. T., Katagiri, Y., Rosenwak, Z., \& Palermo, G. D. (2005). Effect of treating induced mitochondrial damaged on embryonic development and epigenesis. Biology Reproduction, 72, 584-592.

Valojerdi R. M., Yazdi E. P., Karimian L., Hassani F., \& Movaghar B. (2009). Vitrification versus slow freezing gives excellent survival, post warming embryo morphology and pregnancy outcomes for human cleaved embryos. Journal of Assisted Reproduction and Genetics, 26, 347-354.

Van Blerkom, J., Davis, P., \& Alexander, S. (2000). Differential mitochondrial distribution in human pronuclear embryos leads to disproportionate inheritance between blastomeres: Relationship to the microtubular organization, ATP content, and competence. Human Reproduction, 15, 2621-2633

Wennerholm, U. B. (2000). Cryopreservation of embryos and oocytes: Obstetric outcome and health in children. Human Reproduction, 15, 18-25.

Whittingham, D. G., Leibo, S. P., \& Mazur, P. (1972). Survival of mouse embryos frozen to -196 degrees and -269 degrees C. Science, 178, 411-414

Zeng, H.T., Ren, Z., Yeung, W.S., Shu, Y.M., \& Liang, X.Y. (2007). Low mitochondrial DNA and ATP contents contribute to the absence of birefringent spindle imaged with PolScope in in vitro matured human oocytes. Human Reproduction, 22, 1681-1686. 